\title{
La configuración de ejercicios prácticos en clases de fonética española para alumnos de estudios filológicos (consideraciones metodológicas)
}

\author{
The arrangement of practical exercises in Spanish \\ pronunciation classes for modern languages degree students \\ (methodological considerations)
}

\author{
Jerzy Szałek \\ Universidad Adam Mickiewicz de Poznań \\ jerzysz@amu.edu.pl
}

\begin{abstract}
This study is devoted to methodological considerations on the optimization of practical exercises in Spanish pronunciation classes for modern languages degree students. Combining years of experience with the newest theoretical approaches in the area of articulatory phonetics of the Spanish language, the author presents a host of useful advice and practical arrangements, based on a thorough analysis of phonetic contrast between Spanish and Polish.
\end{abstract}

Keywords: articulatory phonetics, Spanish-Polish phonetic contrast, methodology and the arrangement of pronunciation exercises

\section{PRELIMINAR}

La competencia fónica de aprendices de español en diferentes etapas de estudio de esta lengua no ha recibido nunca debida y/o demasiada atención por parte de los profesores de lengua para extranjeros ${ }^{1}$. Es obvio que en los estudios de filología es indispensable adoptar y seguir algunas metodologías teóricas especiales, así como

\footnotetext{
${ }^{1}$ Véanse, por ejemplo, Llorente Pinto (2013).
} 
talleres de ejercicios prácticos con el fin de conseguir lo que sería aceptable para mantener un alto y deseado nivel universitario. La enseñanza de la pronunciación es una tarea muy compleja como tal, pero solo en los estudios filológicos esa tarea ha de considerarse como específica y profesional, lo que supone ante todo la unión de la teoría fonética con la práctica de hablar bien y según la norma escogida. El diplomado en filología española debe pronunciar correctamente, pero también saber por qué y para qué debería hacerlo, cuáles son los objetivos de la fonética como materia auxilar en la enseñanza del español, cuál es la base de articulación de la lengua, los fonemas y los alófonos, el acento, la entonación, la coarticulación, los enlaces, el ritmo, el tempo, etc.

A la hora de aprender y enseñar la fonética española como extranjera hay que conocer su didáctica, saber en qué consiste el contraste entre la fonética vernácula (natal) y la de la lengua meta, lo que es de primordial importancia si se trata de las lenguas parecidas o cercanas fonéticamente como p. ej. el español y el polaco (Szałek 2014: 243-249). Más aún, debería darse cuenta de la diversidad de pronunciaciones normativas en el español moderno. Asimismo, reconocer las diferencias fonéticas generales o particulares en las modalidades del español en Hispanoamérica, lo que es cambio dialectal, variedades diatópicas, diastráticas o diafásicas ${ }^{2}$. En lo que atañe a la diversidad de normas hispánicas y su extensión actual merece la pena recordar sobre todo el trabajo que llevaron a cabo los autores académicos y otros hispanistas al sintetizar la información referente a la manera actual de pronunciar en español en cada uno de los países hispanoamericanos. Nos referimos en este lugar a la primera prueba de unir lo teórico con lo práctico en fonética hispana en la Nueva gramática de la lengua española. Fonética y fonología, RAE, AALE (2011) ${ }^{3}$. El profesor de fonética ha de ser consciente de la necesidad de informar a sus alumnos de las diversas normas fonéticas, es decir, de las variaciones en la pronunciación del español, así como también qué norma va a prevalecer en su clase de fonética. Hay que recordar insistentemente el funcionamiento en el ámbito español de dos subsistemas principales, el llamado seseante (que cambia la $/ \theta /$ en la $/ \mathrm{s} /$ dental) y el minoritario distinguidor que mantiene la pronunciación de $/ \mathrm{s} /$ alveolar y la $/ \theta /$. En Polonia, en los estudios filológicos, en la clase de fonética se parte del estándar

\footnotetext{
${ }^{2}$ Hay que tener en cuenta que no existe una división fonética general entre la variante peninsular del español y la de Hispanoamérica; más bien o más estrictamente hablamos de variantes peninsulares e hispanoamericanas, como tampoco lo decimos del español de América (como se sabe, es mejor y más preciso puntualizar en el español en América).

${ }^{3}$ El tomo que mencionamos contiene un valioso anejo: Las voces del español. Tiempo y espacio en formato DVD que complementa los contenidos teóricos con diversas ramas prácticas, entre las cuales destaca la que permite al profesor de fonética y su alumno encontrar una herramienta de gran valor, es decir, las entonaciones de todo el ámbito hispánico. Como se menciona en el prefacio de la obra, este DVD "permite escuchar las voces y los sonidos del español en su verdadera diversidad en boca de hablantes de todas las zonas de la lengua" (RAE, AALE, 2011: XXIII).
} 
español, pero al mismo tiempo estamos abiertos a las preferencias de nuestros alumnos, ofreciéndoles toda la información sobre las características propias de los dos subsistemas fonéticos del español. Por ejemplo, la mayoría de los aprendices polacos prefiere pronunciar la $/ \mathrm{s} /$ predorsal dental en vez de la /s/ ápicoalveolar, lo que está ligado con un arraigado hábito de articular el sonido dental alveolar /s/ polaco, que se parece más a las variantes predorsales dentales hispanas. A decir verdad, el fonema /s/ ápicoalveolar español pertenece a los sonidos más difíciles de alcanzar por los alumnos polacos, especialmente por su diferente punto de articulación (el ápice de la lengua en la variante polaca no es cóncavo en el contacto con la zona dental alveolar, además es menos tenso en comparación con la variante española $)^{4}$.

\section{ALGO MÁS SOBRE EL CONTRASTE FONÉTICO ESPAÑOL-POLACO}

Como hemos comentado en el apartado anterior, para darse cuenta de las dificultades de alumnos en el aprendizaje de fonética española hay que poseer muchos conocimientos sobre el contraste fonético entre las dos lenguas, el español y el polaco. Es curioso que los métodos de español para alumnos polacos escasean en este tipo de datos limitándose solo a la breve información sobre la correspondencia que hay entre grafemas de ambas lenguas, lo que es insuficiente y digamos sinceramente, reprochable. En este artículo, por falta de espacio, vamos a señalar tan solo algunos de los problemas más importantes, sobre todo, desde el punto de vista de la fonética articulatoria, apoyándonos; sin embargo, en las demás ramas fonéticas. Ahora bien, recordemos que la fonética articulatoria estudia la producción de los sonidos del habla detectando los movimientos de los órganos articulatorios en el llamado aparato fonador. El contraste fonético se observa tanto en el funcionamiento de la base de articulación de las dos lenguas, sus elementos segmentales o sonidos del habla (vocales y consonantes), como también en la mayoría de los demás fenómenos fonéticos, entre los cuales destacan: la coarticulación, la entonación, el ritmo y el tempo. Las características más importantes de la base de articulación española y la polaca las hemos descrito en otra aportación nuestra, de ahí que en este lugar vamos a prestar más atención al contraste particular de algunos fenómenos específicos de las dos fonéticas ${ }^{5}$. A la hora de aprender la pronunciación del español moderno hay que indicar, en primer lugar, la disposición propia del castellano que consiste en un alto grado de tensión articulatoria superior a la del polaco. El que

\footnotetext{
${ }^{4}$ Como señala Vaquero de Ramírez (1996: 34-35) la /s/ en Hispanoamérica es polimórfica y ya Canfield (1962) no dejó de registrar hasta cuatro de sus variantes.

${ }^{5}$ Véanse al respecto Szałek (2015), donde hemos presentado la característica de la base de articulación general a la luz de sus rasgos fundamentales, así como algunas observaciones contrastivas sobre los ajustes articulatorios no localizados y los localizados en español y polaco.
} 
comienza a hablar en español ha de tener presente que los hispanohablantes suelen mantener algo más tensa la musculatura de las mejillas, lo que quiere decir que el relajamiento de la pronunciación tanto de las vocales como de las consonantes españolas puede cambiar su timbre y/o la entonación, así como también el ritmo de la cadena hablada. La pronunciada tensión articulatoria en español no influye en el alargamiento de los segmentos vocálicos que no cambian su brevedad. De este modo, los que desean hablar correctamente en español deben evitar la reducción de sus vocales, especialmente en las átonas, lo que supone algún esfuerzo adicional para los estudiantes polacos que, por lo general, están acostumbrados a su reducción bajo la influencia del aprendizaje de otras lenguas extranjeras que estudian o que han estudiado, como p. ej. el francés o el inglés. Recordemos, asimismo, que la base de articulación polaca se caracteriza por el movimiento menos tenso de la lengua, de los labios y del velo del paladar, el redondeamiento de las $[\mathrm{o}]$ y [u] y la cerrazón de las [i] y [e] poco marcadas. En contraposición al polaco, la base de articulación española no debilita los segmentos en posición implosiva de fin de palabra (Nowikow, Szałek 2011: 22-23; Szałek 2015: 148-149).

Si hablamos de un verdadero contraste fonético entre el español y el polaco hay que mencionar sobre todo la principal y bien conocida especialidad española de los alófonos aproximantes $[\beta, \delta, \gamma]$ de los fonemas $/ b, \mathrm{~d}, \mathrm{~g} /$ que el polaco sencillamente desconoce. En la lengua polaca existen solo equivalentes oclusivos de los fonemas /b, d, g/, mientras que, por otra parte, esos fonemas disponen en polaco también de variantes palatizadas que por interferencia están influyendo negativamente en una correcta pronunciación de los equivalentes oclusivos españoles por los estudiantes polacos (cf. p. ej. las palabras polacas: [d,jádem] diadem, [g,jérki] gierki, [b,jáwi] biały). Para dar un ejemplo, el alófono polaco palatizado $[b$,$] es oclusivo bilabial$ sonoro, pero su articulación difiere de su homólogo [b]. A la hora de su articulación, la lengua cambia la posición plana y su mediodorso se acerca más al paldar duro (Dyszak, Laskowska, Żar-Święcicka 1997: 63-68). La palatización polaca interfiere en muchos casos en la correcta emisión de los sonidos españoles, de ahí que el timbre suele cambiar en la mayoría de los casos en combinaciones de las secuencias fónicas españolas del tipo [ni] o [li], p. ej. en: [líma] lima, [límite] límite, [líriko] lírico, [lítro] litro; [níco] nicho, [níno] niño, [aßaníko] abanico, etc.

La correcta pronunciación de este tipo de combinaciones supone la aplicación en clase de fonética de muchos ejercicios antipalatizantes de contraste.

Volviendo a las aproximantes españolas $[\beta, \delta, \gamma]$, que como hemos señalado antes, constituyen un rasgo propio y típico del español, es indispensable darse cuenta de su modo y lugar de articulación. Al explicar la articulación de la problemática aproximante bilabial sonora $[\beta]$ no podemos dejar de mencionar que el movimiento de los labios en su caso es distinto en comparación con las cercanos alófonos polacos, el oclusivo bilabial sonoro [b] y el fricativo labiodental sonoro [v]. Este último no aparece en español. En la práctica del auditorio tanto los docentes como 
los discentes polacos tienen un gran problema a la hora de reconocer la verdadera naturaleza fónica de la variante española $[\beta]$, que es distinta de la de las dos variantes polacas. En uno de nuestros estudios anteriores (Szałek 2015: 153) explicamos detalladamente el mecanismo de su producción, es decir, para pronunciar adecuadamente la aproximante $[\beta]$ española hay que tratar de adelantar un poco el labio inferior para que no toque los incisivos superiores controlando la abertura de la boca, es decir, una posición "sin oclusión", así como manteniendo la tensión articulatoria adecuada. Al mismo tiempo, es preciso ensanchar un poco la boca tensando los músculos de las mejillas y los labios sin cambiar la duración y la intensidad. Los labios se aproximan entre sí sin cerrarse del todo. Como confirma Martínez Celdrán (2013: 262-263) “(...) El grado de abertura es variable por lo que resulta ser una categoría no discreta, puede ir desde un cierre no completo hasta la abertura casi vocálica (...)". En conclusión, sería recomendable poner más fuerza en la sílaba acentuada de la palabra, para mantener su tensión. La aproximante $[\beta]$ aparece en posición central de la palabra e intermedia entre palabras, p. ej.: [laßoráßle] laborable, [éntreßákas] entre vacas, etc. (no aparece al principio de la palabra y después de las nasales). Hay que subrayar que su dominio requiere muchos ejercicios, entre los cuales, los de contraste con sus variantes polacas antes mencionadas (Szałek 2015: 153).

No podemos perder de vista tampoco, otro tipo de contraste que hay entre las dos fonéticas en cuestión. En algunas de nuestras aportaciones anteriores hemos descrito detalladamente el contraste con la llamada "troika castellana", es decir, las tres consonates más características del español frente a las de las demás lenguas del entorno europeo, $/ \overline{\mathrm{r}} / \mathrm{x} / \mathrm{y} / \mathrm{s} /$ (Szałek 2012, 2014a, 2018). Los tres fonemas difieren considerablemente de sus variantes más cercanas polacas. La oposición entre la vibrante alveolar sonora $[\bar{r}]$ y la percusiva alveolar sonora $[\mathrm{r}]$ no existe prácticamente en otras lenguas europeas, inclusive la polaca. La variante polaca $/ \mathrm{r} /$, que es más cercana en cierto modo a la percusiva española, constituye un obstáculo para que nuestro alumno pueda conseguir fácilmente la pronunciación de la vibrante española $[\bar{r}]$ que es múltiple, y de este modo, requiere un esfuerzo articulatorio adicional de acuerdo con su propia naturaleza, que se debe al efecto físico de Bernoulli (se trata de un ciclo de dos o más oclusiones que fortalecen la vibración) (Martínez Celdrán, Fernández Planas 2007: 149-151). El acercamiento articulatorio de la /s/ polaca (como p. ej. en la palabra polaca [sósna] sosna (= esp. "pino") que es fricativa, predorsal-dental, sorda, no palatizada (no aparece delante de [i] $)^{6}$ con la española predorsal dental [s], hace que nuestros estudiantes no estén dispuestos a adquirir con facilidad la típica variante castellana de [s] , que les parece un poco "incómoda", algo "artificial" y difícil de alcanzar sin un esfuerzo adicional. Desde hace algún tiempo y siguiendo las recomendaciones académicas, no insistimos

\footnotetext{
${ }^{6}$ El polaco, aparte de la [s] dura, dispone también de las variantes palatizada [s'] y blanda [ṣ], respectivamente.
} 
actualmente para que la consigan a toda costa, teniendo en cuenta que la mayoría de hispanohablantes pronuncian las variantes predorsales en vez de la alveolar castellana. En cuanto a la [x] española, hay que observar que en la mayoría de los casos su adquisición no resulta problemática, aunque su correcta pronunciación delante de las vocales $[\mathrm{a}, \mathrm{u}, \mathrm{o}]$ requiere también de varios ejercicios de contraste (porque la posición uvular española no corresponde exactamente a la emisión velar polaca).

\section{PROPUESTA METODOLÓGICA DE TIPOLOGÍA}

Dándonos cuenta del contraste que hemos esbozado arriba, vamos a presentar en este lugar una propuesta de ejercicios que en nuestra opinión son recomendables e indispensables para salvar algunas de las dificultades fonéticas de estudiantes universitarios polacos. Nuestras consideraciones metodológicas las apoyamos en nuestra larga experiencia de impartir clases de fonética en este nivel de estudios. En primer lugar, refiriéndonos al triángulo vocálico del español actual, insistimos en el aumento de la tensión articulatoria $(1)^{7}$. Las vocales españolas de las sílabas preponderamente abiertas son más tensas que las polacas, lo que no quiere decir que haga falta cambiar su duración. Para fines didácticos suponemos que las vocales de ambas lenguas son cortas sin reducción alguna de las átonas. El aumento de la tensión consiste tan solo en poner más fuerza articulatoria, lo que conseguimos gracias al reforzamiento acentual dentro de la sílaba y, al mismo tiempo, venciendo el relajamiento en el movimiento de los labios que, como hemos mencionado, están menos tensos en polaco (cf. p. ej. el redondeamiento de la $[\mathrm{o}] \mathrm{y}[\mathrm{u}]$ en diferentes posiciones). La tensión más fuerte influye considerablemente en una pronunciación más expresiva. Los ejercicios fonéticos que cambian progresivamente la posición de la vocal en la palabra (2) son los más adecuados ${ }^{8}$. La aplicación de ejercicios de contraste antipalatizantes (3), que ya hemos señalado en el apartado 2, parece imprescindible también desde la primera clase de fonética dedicada a las vocales. Asimismo, sería preciso marcar más el movimiento del postdorso de la lengua cuando pronunciamos las vocales españolas posteriores $[\mathrm{u}]$ y $[\mathrm{o}]$. La detenida observación de la emisión de las róticas españolas (4) por los alumnos polacos ha de tener preferencia no solamente en lo que atañe a la misma oposición entre la $[\mathrm{r}]$ y la $[\overline{\mathrm{r}}]$, respectivamente ([péro] / [per̄o] pero / perro). Hay que configurar toda una serie de ejercicios que tomen en consideración tanto los lugares de su desaparición (en principio de palabra, donde aparece solo la [ $\overline{\mathrm{r}}]$ ) como los efectos de neutralización en posición implosiva y final de palabra (archifonema /R/ (p. ej.: [árma] = [árma] = [á.rma], arma, $[$ tomár $]=[$ tomar $]=[$ tomar $]$, tomar, etc.). Los ejercicios de contraste $(5)$ son

\footnotetext{
${ }^{7}$ Nuestras recomendaciones las señalamos en este apartado subrayadas y con números arábigos.

${ }^{8}$ Véanse, por ejemplo, la configuración de ejercicios de vocales propuesta en Nowikow (2012 [1992]: 22-25).
} 
muy provechosos (p. ej. [rurúl] rural, [retráto] retrato, [bárera] barrera, etc.), pero no se debe olvidar tampoco el fenómeno de la coarticulación (asimilación) en las secuencias del tipo [dō̄wé $\delta a s]$ dos ruedas, [loríkos] los ricos u otros (p. ej. cuando la rótica aparece después de las consonantes alveolares $/ 1 /, / \mathrm{n} /, / \mathrm{s} /:$ [ónra] honra, [elrío] el río, etc.). En este caso, sería recomendable también hacer una serie de ejercicios con trabalenguas (6), p. ej.: El perro de San Roque no tiene rabo, porque Ramón Rodríguez se lo ha robado; Rosa Rizo reza en ruso, en ruso reza Rosa Rizo; Erre con erre guitarra, erre con erre carril, ruedan y ruedan las ruedas del ferrocarril, etc.

Los ejercicios prácticos con las róticas se deben hacer empezando por las posiciones intermedias intervocálicas, debido al cambio en el punto de articulación y el efecto de Bernouli en el caso de la $[\bar{r}]$. De la misma manera intermedia hay que trabajar al ejercitar las aproximantes españolas $[\beta],[\delta]$ y $[\gamma]$ (7): [páßa] pava, [úßa] uva, [nwéßo] nuevo, [núße] nube, [njéße], etc. La aproximante dental sonora española $[\delta]$ no hay que confundirla con la fricativa dental sonora inglesa $[\delta]$. Tampoco se puede realizar la $[\delta]$ en posición absoluta de palabra como la [t] polaca

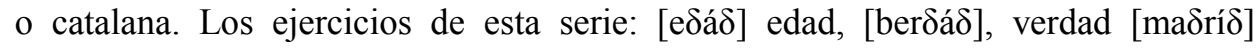
Madrid, etc. requieren de nuestros discentes mucha atención a la hora de situar la lengua en el borde de los incisivos inferiores.

\section{A MODO DE CONCLUSIONES}

En esta aportación tratábamos de añadir algunas de nuestras experiencias didácticas en la tarea de enseñar la fonética del español actual a los estudiantes universitarios polacos. La extensión del artículo no permite ampliar la información sobre muchos más casos específicos que ha de tener en consideración el profesor de fonética a la hora de impartir sus clases de esta materia. Hay que tener presente que tampoco los segmentos no problemáticos de las dos lenguas, la española y la polaca, pueden coincidir en cuanto a algunos rasgos fonéticos. La pronunciación de la [t] española, para dar un ejemplo, no coincide tanto con la pronunciación de la [t] inglesa como la de la [t] polaca. En este caso, si no bajamos la lengua a los dientes, el timbre premanece sin cambio. Para darse cuenta de la complejidad de casos específicos, merece la pena observar la pronunciación española de la oclusiva dental sorda [t] en coda silábica frente a la polaca [t]: (esp.) [adléta] atleta y (pol.) [atléta] atleta. Como confirma Martínez Celdrán (2013: 270-271), la sonorización de la [t] en posición implosiva se realiza tan solo en la variante peninsular, mientras que en Hispanoamérica por lo general se pronuncia como sonido sordo (igual que en polaco). "El conjunto -tl-, dice Marínez Celdrán (2013: 270), pasa de ser heterosilábico en la Península a ser tautosilábico en Latinoamérica".

De este modo, la configuración de ejercicios de fonética en el ámbito de estudios filológicos resulta a fin de cuentas muy importante y hasta decisivo para conseguir el éxito en el aprendizaje de una verdadera base de articulación española. 


\section{BIBLIOGRAFÍA}

Canfield, D. (1962). La pronunciación del español en América. Bogotá: Instituto Caro y Cuervo.

Dyszak, A., Laskowska, E., Żak-Święcicka, M. (1997). Fonetyczny i fonologiczny opis współczesnej polszczyzny. Bydgoszcz: Wydawnictwo Wyższej Szkoły Pedagogicznej.

Llorente Pinto, M. del R. (2013). La importancia de la enseñanza de la pronunciación In M.A. Penas Ibáñez (ed.), Panorama de la fonética española actual (pp. 229-252). Madrid: Arco/Libros.

Martínez Celdrán, E. (2013). Los sonidos obstrueyentes en la cadena hablada In M.A. Penas Ibáñez (ed.), Panorama de la fonética actual (pp. 253-289). Madrid: Arco/Libros.

Martínez Celdrán, E., Fernández Planas, A.M. (2007). Manual de fonética española. Articulaciones y sonidos del español. Barcelona: Ariel Lingüística.

Nowikow, W. (2012 [1992]). Fonetyka hiszpańska. Warszawa: Wydawnictwo Naukowe PWN.

Nowikow, W., Szałek, J. (2001). Introducción a la fonología y la fonética españolas. Poznań: Wydawnictwo Naukowe UAM.

Penas Ibáñes, M.A. (ed.) (2013). Panorama de la fonética española actual. Madrid: Arco/Libros.

Szałek, J. (2012). La "troika fonética castellana" o trío de fonemas castellanos más típicos. Neophilologica, 24, 219-226.

Szałek, J. (2014a). Las consonantes róticas españolas a la luz de las últimas investigaciones fonológicas, fonético-articulatorias y acústicas. In Z. Bułat Silva, M. Głowicka, J. Wesoła (eds.), Variación, contraste, circulación. Perspectivas lingüisticas en el hispanismo actual (pp. 21-28). Wrocław: Wydawnictwo Uniwersytetu Wrocławskiego.

Szałek, J. (2014b). Criterios de diseño de una didáctica fonética del español para profesores de lengua polacos. In J. Pawlik, J. Szałek (eds.), Lingüistica española en Polonia: líneas de investigación (pp. 243-249). Poznań: Wydawnictwo Naukowe UAM.

Szałek, J. (2015). ¿Cómo adquirir con más facilidad la base de articulación española? Entre la teoría y y la práctica. Itineriarios. Revista de estudios lingüisticos, literarios, históricos $y$ antropológicos, 22, 143-155.

Szałek, J. (2018). Apuntes teóricos y prácticos sobre la enseñanza del fonema fricativo velar sordo /x/ a nivel de estudios universitarios. In J. Bień, B. Brzozowska-Zburzyńska, A.M. López González, W. Nowikow (eds.), Lingüistica en Polonia: tendencias y direcciones de investigación (pp. 283-294). Łódź/Lublin: Wydawnictwo Uniwersytetu Łódzkiego.

Vaquero de Ramírez, M. (1996). El español de América I. Pronunciación. Madrid: Arco Libros. 